\title{
Recurrent Leiomyomatosis Peritonealis Disseminata: Sonographic and Laparoscopic Correlation
}

\author{
Ulrich Honemeyer, J ason R Ross, J ami J Barnard, Kallie Appleton, Thomas G Tullius J r \\ Lisa Montgomery, Sanja Kupesic Plavsic
}

\begin{abstract}
We present a 39-year-old GOPO with history of infertility treatment-caused ovarian hyperstimulation and uterine fibroids, who was found to have a rare pathological presentation. Peritoneal lesions were identified during laparoscopic treatment for this patient's fibroids. Histological evaluation of these lesions revealed leiomyomatosis peritonealis disseminata (LPD), also known as disseminated peritoneal leiomyomatosis. While LPD can often be confused for metastatic leiomyosarcoma due to the histological presence of fibroblasts, myofibroblasts, smooth muscle, collagen, and spindle cell appearance, cases of LPD are generally benign, and present no need for therapy in asymptomatic cases. We present a discussion based on the clinical and sonographic presentation of LPD, its pathophysiology, treatment options and prognosis. A question set has been designed to reinforce comprehension of this presentation and associated topics for health care professionals.
\end{abstract}

Keywords: Leiomyomatosis peritonealis disseminata, Disseminated peritoneal leiomyomatosis, Metastatic leiomyosarcoma, Spindle-cells, Fibroids, Infertility, Ovarian hyperstimulation.

How to cite this article: Honemeyer U, Ross J R, Barnard J J , Appleton K, Tullius TG J r, Montgomery L, P lavsic SK. Recurrent Leiomyomatosis Peritonealis Disseminata: Sonographic and Laparoscopic Correlation. Donald School J Ultrasound Obstet Gynecol 2012;6(3):327-332.

\section{Source of support Nil}

Conflict of interest: None declared

\section{CASE REPORT}

A 39-year-old GOP0, Caucasian female was referred for management of primary infertility, as she had previously undergone several cycles of controlled ovarian hyperstimulation using gonadotropin releasing hormone $(\mathrm{GnRH})$ analogs and follicle stimulating hormone (FSH) without achieving pregnancy. Diagnosis of uterine fibroids was made through sonographic evaluation (Figs 1 and 2), and she was subsequently scheduled for laparoscopic myomectomy.

Upon laparoscopy, the patient was found to have disseminated peritoneal lesions, in addition to the multiple uterine fibroids (Fig. 3). Biopsies of the peritoneal, omental, intestinal and liver lesions were obtained at that time (Figs 4 to 6).

Histological evaluation of the peritoneal lesions yielded a cellular composition similar to leiomyomata, with uniform, spindle-shaped smooth muscle cells in a whorled arrangement, a low mitotic index and no pleomorphism.

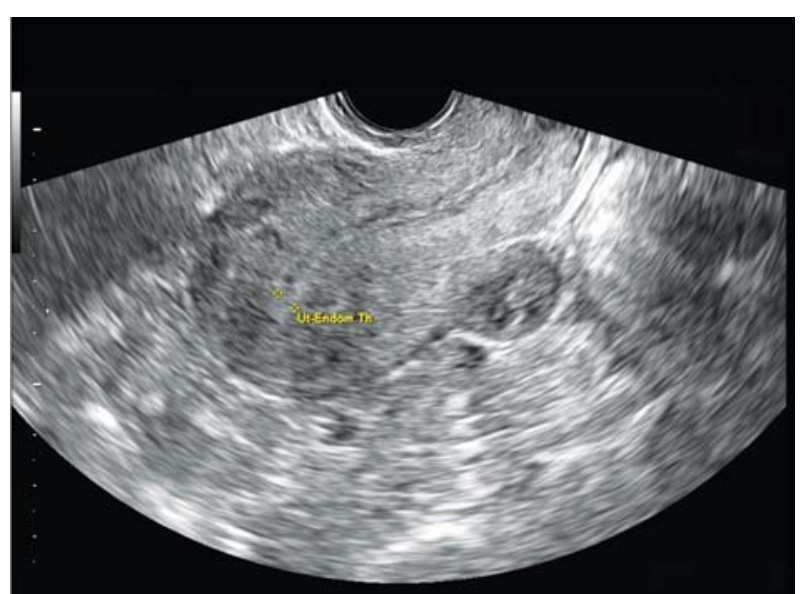

Fig. 1: Transvaginal ultrasound of the uterus. Note irregularly shaped uterus with evidence of subserosal uterine tumor

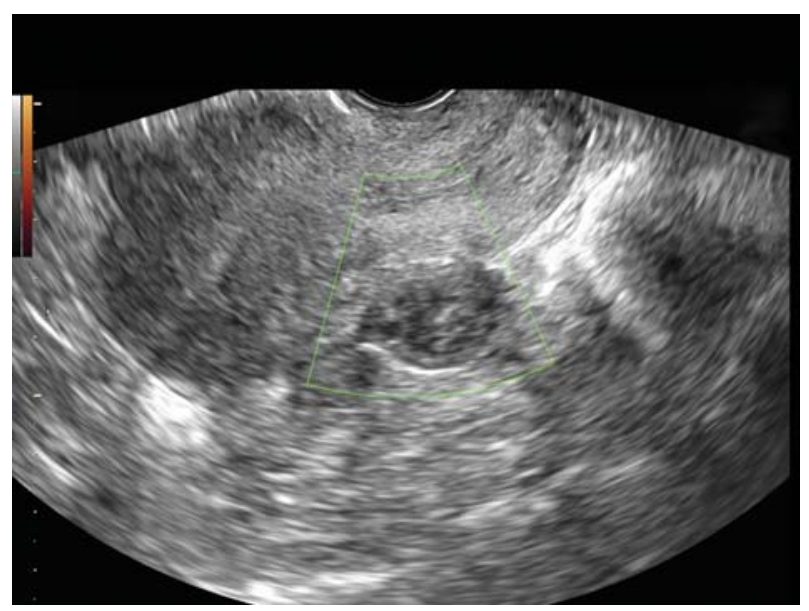

Fig. 2: Color Doppler image of the same patient. Note absence of tumoral/peritumoral vascularization

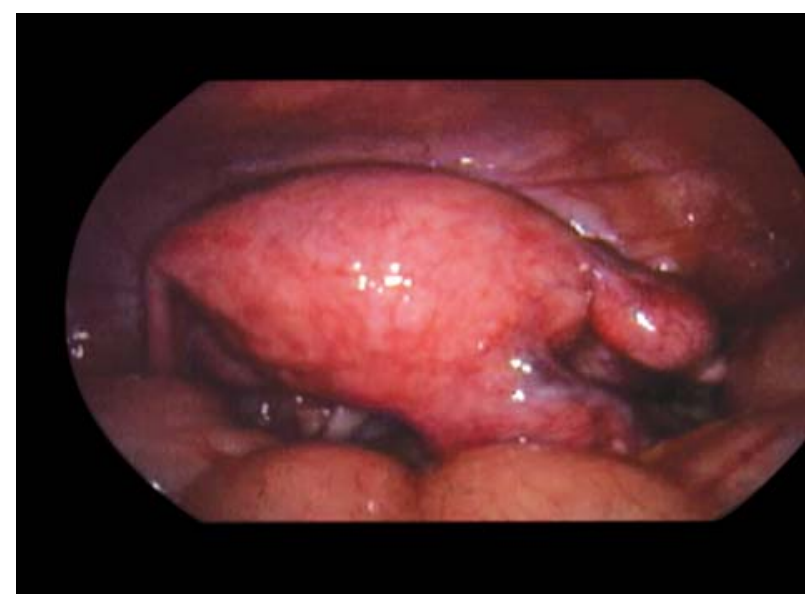

Fig. 3: Laparoscopic image of the uterus in a patient with LPD 


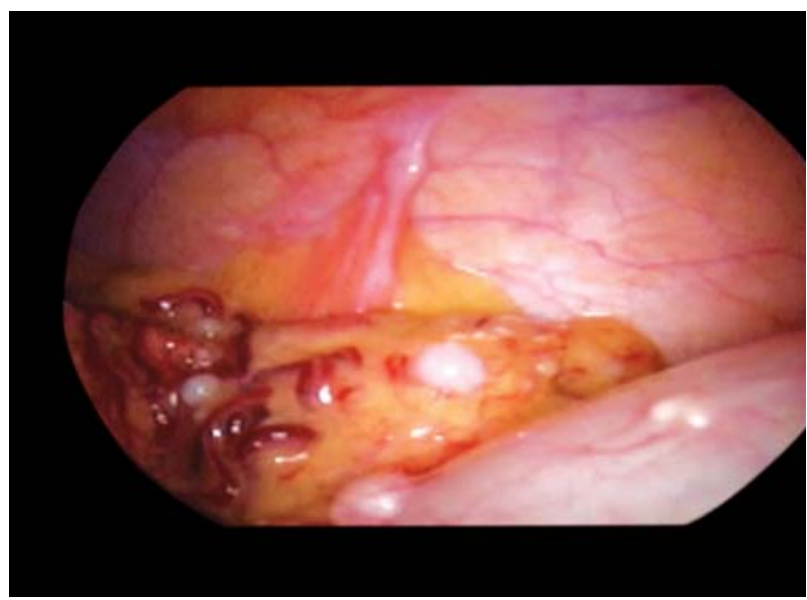

Fig. 4: Laparoscopic image of the omental, peritoneal and intestinal dissemination of LPD

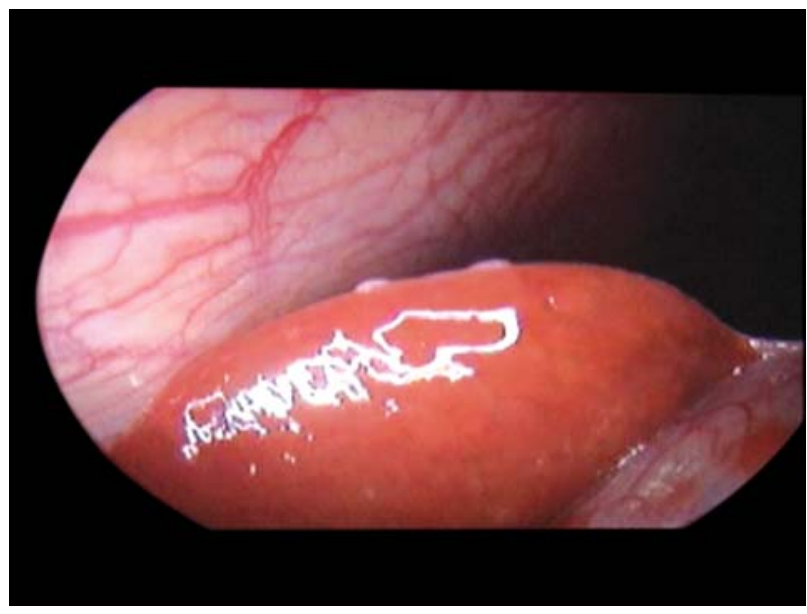

Fig. 5: Right liver lobe of the same patient

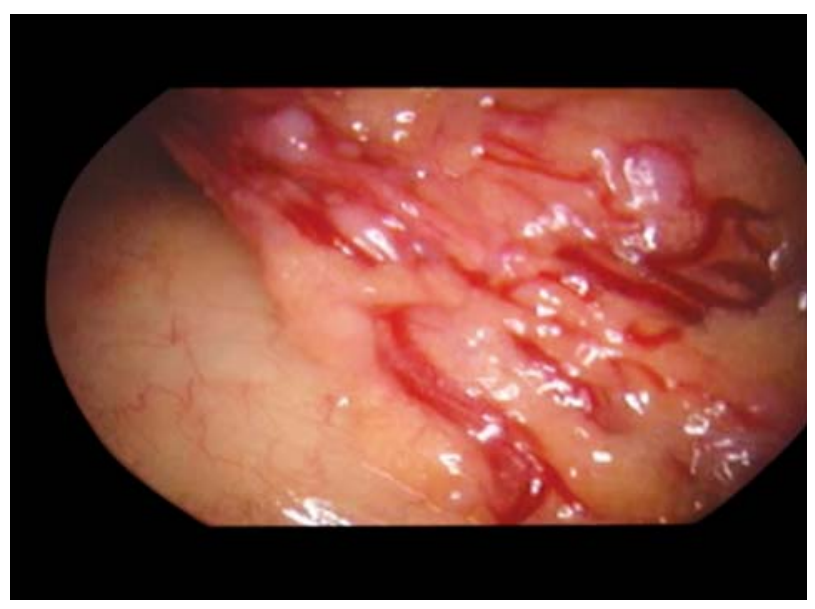

Fig. 6: O mental spread of LPD

Immunohistochemically, the intravascular parts of the tumors showed a positive staining reaction for $1 \mathrm{~A} 4, \mathrm{H} \mathrm{HF} 35$, vimentin and desmin. Estrogen and progesterone receptors were also positive. Final diagnosis was confirmed as leiomyomatosis peritoneal is disseminata (LPD), otherwise known as disseminated peritoneal leiomyomatosis. ${ }^{1}$
A fter laparoscopy and histological verification of LPD, the patient received monthly $\mathrm{GnRH}$-agonist injections (Decapeptyl 3.75 mg Depot, Ferring, Kiel, Germany) for 3 months. A fter $\mathrm{GnRH}$ agonist treatment, second-look laparoscopy was planned; however, the patient opted against undergoing the procedure. She was advised to avoid infertility treatments and pregnancy in the future because of the potential for excessive endogenous progesterone and estrogen levels fostering the progression of LPD.

Despite her physician's recommendation, the patient became pregnant with reproduction assistance 3 years after the laparoscopic and hormonal treatment, and she delivered a heal thy baby by lower segment cesarean section (LSCS). At the time of LSCS, intraoperative inspection of the abdomen reveal ed leiomy omatosis peritoneal is disseminata, albeit less extensive than at the time of original diagnosis.

\section{DISCUSSION}

LPD, also known as diffuse peritoneal leiomyomatosis (DPL), is a rare condition in which multiple smooth muscle or smooth muscle-like nodules develop anywhere in the subperitoneal abdominal cavity. These nodules, though histologically benign, cannot reliably be macroscopically distinguished from peritoneal carcinomatosis. Smooth muscle cell metaplasia of the subperitoneal mesenchyme is considered the cause of LPD. ${ }^{2} \mathrm{Had}$ the pathology extended to the lymph nodes or intrathoracic cavity, then histologically similar pathologies, such as benign metastasizing leiomyoma and lymphangioleiomyomatosis, may have been considered. A s the histological picture of LPD is a spindle cell dominated mix of fibroblasts, myofibroblasts, smooth muscle and collagen, the spindle cell dominance can cause LPD to be mistaken for metastatic leiomyosarcoma; however, the metastatic nodules of leiomyosarcoma are generally larger than $1 \mathrm{~cm}$, locally invasive and fewer in number. ${ }^{1} \mathrm{~A}$ Iso, hel ping to differentiate the two is pleomorphism and a mitotic index greater than 10, which are characteristics of leiomyosarcoma unshared by LPD.

Although LPD mostly affects women of reproductive age, cases have also been reported in men with limited or no estrogen and progesterone receptor activity. ${ }^{3}$ L PD seems to be associated with exposure to elevated exogenous and endogenous female gonadal steroid levels, which may occur with hormonally active ovarian tumors, pregnancy, gonadotropin therapy/in vitro fertilization (IVF), or even tamoxifen use. In this respect, LPD appears to behave similarly to uterine leiomyomata. ${ }^{4-6}$ LPD cases are generally considered benign; however, progression to malignancy has been reported. ${ }^{3,7,8}$ M ost cases of LPD are incidentally discovered, and for asymptomatic patients, there may be no need for therapy. For symptomatic patients, or those with 
worrisome gross or histopathological features, bilateral salpingo-oophorectomy can often be a definitive cure due to the tumor's estrogen dependence. The observation that LPD can be triggered by increased gonadotropin levels suggests an option of conservative treatment of LPD with $\mathrm{GnRH}$ agonists, which has reportedly been used with success. ${ }^{9}$ In young patients or those desiring to maintain fertility, the ovaries may be preserved with a regimen of $\mathrm{GnRH}$ analogs applied for a total of 3 to 6 months. We followed these recommendations by administering a $\mathrm{GnRH}$ course of 3 months duration after initial diagnosis, but unfortunately, were limited in analyzing the therapeutic outcome of that regimen due to patient's refusal of a second-look laparoscopy. While the pregnancy achieved 3 years after initial LPD diagnosis fulfilled the patient's greatest wish, the persistence of LPD became obvious when the abdomen was inspected during LSCS. LPD was confirmed by histological examination. The effectiveness of our therapeutic trial is uncertain in this case as is the course of the patient's pathological progression, especially considering her experiencing the elevated estrogen state of pregnancy. Despite the possibility of treatment failure in this case, even surgical castration and excision of the lesions, which is considered definitive therapy, has been associated with LPD recurrence. ${ }^{10}$

\section{SELF-ASSESSMENT QUIZ}

1. M s. Jackson, a 34-year-old G3P2 A 1 presents with symptoms of menorrhagia. Her uterus is enlarged and on palpation there are no adnexal masses. Ms. $J$ ackson's luteal phase progesterone level is calculated to be $17 \mathrm{ng} / \mathrm{ml}$ (normal $4.4-28.0 \mathrm{ng} / \mathrm{ml}$ ). Which of the following is the least likely cause of her menorrhagia?
A. Adenomyosis
B. Hypothyroidism
C. Uterine leiomyomata
D. Uterine polyp

2. Your 29-year-old patient has been attempting to achieve a pregnancy for the past 16 months. Her husband of 2 years has a child from a previous relationship and seems to be in good health. Y our patient has never been pregnant before and has always had very regular, although heavy periods. U pon questioning, she reports that she is bleeding in clots. There are no abnormal vaginal findings, but a slightly enlarged uterus is discovered on pelvic examination. If a diagnosis of infertility secondary to uterine leiomyomatosis is made, what type of fibroid(s) is most likely involved?
A. Intramural
B. Subserosal
C. Cervical
D. Submucosal

3. A 56-year-old, G5 P5, presents with postmenopausal bleeding and enlarged uterus. Which of the following is the WORST option for management?
A. Pelvic ultrasound
B. Endometrial biopsy
C. Watchful waiting

D. CT of the abdomen and pelvis

E. Hysterectomy and bilateral sal pingo-oophorectomy

4. Y our 28-year-old patient, GOPO has abdominal CT scans following a motor vehicle collision that demonstrate multiple intra-abdominal nodules. These nodules are identical in appearance to two nodules identified within the uterine walls. Which of the following is the most likely diagnosis?
A. M etastasizing leiomyosarcoma
B. Ovarian endometriosis
C. Spread of the ovarian carcinoma
D. Disseminated intraperitoneal leiomyomatosis

5. A patient is referred to you with a rare past medical history of leiomyomatosis with subsequent $\mathrm{GnRH}$ therapy and ovarian preservation (because at the time of presentation with symptoms of fibroids, the patient still desired maintaining her childbearing capacity). A ccording to her chart, her therapy included taking $\mathrm{GnRH}$ therapeutically for 6 months almost 20 years ago. Standing before you is this now 55-year-old G3P3, who experienced menopause 5 years ago with a loss of height and degree of kyphosis gradually worsening over the past 3 years. What is the correlation/ association between $\mathrm{GnRH}$ and osteoporosis?
A. There is no association
B. A fter the cessation of the drug there is no additional risk for osteoporosis
C. There is no risk of osteoporosis with $\mathrm{GnRH}$ therapy. However, GnRH therapy is associated with osteoarthritis.
D. Women taking $\mathrm{GnRH}$ therapy in their 20 s and 30 s have a higher risk of osteoporosis later in life.


6. A 37-year-old, G3P3 patient with leiomyomatosis has undergone initial GnRH therapy for 6 months. She is currently in your office for routine follow-up 1 month after the cessation of a 6 month therapeutic course of $\mathrm{GnRH}$ therapy. She has done research on the internet regarding her condition and wants your opinion of her long-term prognosis. According to your research, her expected prognosis includes which of the following?
A. Life expectancy of 5 years
B. Infertility
C. Return to normal menstrual cycles
D. Preservation of fertility and return to normal menstrual cycles

7. A G3P3, 37-year-old patient, with a rare pathology of uterine leiomyomatosis, has undergone initial therapy with GnRH for the past 6 months and is currently in your office for routine follow-up. She now wants to know what the next step in treatment is as you transition to long-term therapy.
A. Watchful waiting
B. Surgical castration
C. Surgical debulking
D. All of the above

8. A G3P3, 31-year-old patient, with a rare pathology of uterine leiomyomatosis, has undergone initial therapy with $\mathrm{GnRH}$ for the past 6 months, and is currently in your office for routine follow-up. She now wants to know what the next step in treatment is as you transition to long-term therapy. Y ou discuss her options and together decide that treatment with tamoxifen may be best for her. What are the major box warnings of tamoxifen therapy?
A. Thromboembolic events/gynecologic effects and malignancies
B. Thromboembolic events/ocular effects
C. Gynecologic effects and malignancies/hepato- toxicity
D. Bone marrow suppression/gynecologic effects and malignancies

9. A 39-year-old GOPO, Caucasian female was referred for management of primary infertility, as she had previously undergone several cycles of controlled ovarian hyperstimulation using follitropin beta, decapeptide ganirelix, and dydrogesterone without achieving pregnancy. Diagnosis of uterine fibroids was made through sonographic evaluation, and she was subsequently scheduled for laparoscopic myomectomy. Upon laparoscopy, the patient was found to have disseminated peritoneal lesions, in addition to the multipleuterine fibroids, and biopsies of the peritoneal lesions were obtained at that time. Histological evaluation of the peritoneal lesions yielded a cellular composition similar to leiomyomata, with uniform, spindle-shaped smooth muscle cells in a whorled arrangement, a mitotic index of 23, and mild pleomorphism. What is the next course of action?
A. Watchful waiting
B. LN biopsy
C. Hysterectomy
D. Oophorectomy

10. Your patient has abdominal CT scans following a motor vehicle collision. While no intra-abdominal trauma is visualized, multiple intra-abdominal nodules are incidentally discovered. These nodules are identical in appearance to two nodules identified within the uterine walls and a solitary lung lesion. Which of the following is the most likely diagnosis?
A. Benign metastasizing leiomyomata
B. Uncomplicated uterine fibroids
C. Dissemination of endometrial carcinoma
D. Disseminated peritoneal leiomyomatosis

\section{Correct Ans wers}

1: $B ; 2: D ; 3: C ; 4: D ; 5: D ; 6: D ; 7: D ; 8: A ; 9: B ; 10: A$

1. A nswer: $B$

Explanation: A luteal progesterone value of $17 \mathrm{ng} / \mathrm{ml}$ indicates that $\mathrm{M} \mathrm{s} \mathrm{J}$ ackson is indeed ovulating. Causes of abnormal uterine bleeding in ovulating women are usually anatomical in origin. For example, uterine leiomyomata, uterine polyps, uterine or cervical neoplasms and adenomyosis can all cause menorrhagia. Other possibilities are infections and uterine foreign bodies (for example Paragard ${ }^{\circledR}$ IUDS). Endocrine issues, such as hypothyroidism usually cause menorrhagia by dysregulation of ovulation (thus causing anovulation) via hormonal effects (choice B).

2. A nswer: D

Explanation: Submucosal leiomyomata are located beneath the endometrium and may distort the uterine cavity. Even small lesions in the submucosal layer of the uterus may lead to bleeding and infertility due to uterine cavity distortion (choice D).

3. Answer: $\mathrm{C}$

Explanation: Watchful waiting is not an option for a postmenopausal patient with postmenopausal bleeding and enlarged uterus (choice $\mathrm{C}$ ). The leiomyoma is a monoclonal tumor that is dependent on estrogen and progesterone. Consequently, they should shrink during menopause as the estrogen levels decline. A s the patient is postmenopausal and symptomatic the patient should be actively assessed and treated. 
Recurrent Leiomyomatosis Peritonealis Disseminata: Sonographic and Laparoscopic Correlation

4. Answer: D

Explanation: In disseminated intraperitoneal leiomyomatosis, leiomyomata grow diffusely on the peritoneal and omental surfaces, with uterine lesions serving as their primary source (choice D). It is less likely that the reproductive age patient is presenting with uterine leiomyosarcoma. Since, the nodules have identical appearance to uterine tumors, it is less likely that the patient has ovarian lesions.

5. Answer: D

Explanation: The initial side-effects of $\mathrm{GRH}$ therapy include anovulation, vaginitis and headache. M ost causes of anovulation accompany decreased bone density; however, anovulation does not cause osteoporosis directly. Osteoporosis is usually diagnosed after the 5th decade of life, and is the cumulative effect of gradual bone demineralization. A novulation during the patient's $20^{\prime} s$ and 30 's predisposed her to earlier and more severe osteoporotic changes due to decreased bone density reserve. Estrogen associated with ovulation is protective to bone density reserve (choice D).

6. Answser: D

Explanation: Due to the rarity of the condition, there is low power to most studies concerning leiomyomatosis. Regarding prognosis, the general consensus is that it is reasonable to expect a return to regular menstrual cycles and fertility after conservative therapy (choice D). The actual outcome is dependent on longterm follow-up, the patient's initial health and fertility, and the areas of disease dissemination.

7. Answer: D

Explanation: A ll of the above treatments are acceptable treatments of uterine leiomyomatosis depending on the severity of the diseaseand the patient's desires (choiceD).

8. Answer: A

Explanation: The box warnings of tamoxifen are risks of thromboembolic events/gynecologic effects and malignancies (choiceA). The rest of theansw er choices are rare but serious complications of tamoxifen use. Patient education and monitoring with tamoxifen use should includethefollowing: Lipid profileoptimization, eval uation of any abnormal uterinebleeding, $\mathrm{M} \mathrm{I/stroke}$ education, education on the signs and symptoms of hepatotoxicity, education regarding worrisome ocular changes and monitoring for pancy topenia/neutropenia. There is al so a rare association of tamoxifen usage with uterine sarcoma.

9. Answer: B

Explanation: There is very limited evidence to guide the evaluation of smooth muscle tumors of uncertain malignant potential. An investigation that included imaging or limited LN biopsy would be more appropriate than radical surgery or watchful waiting (choice B).

10. Answer: A

Explanation: Lymph node and/or lung lesions are a feature of benign metastasizing leiomyomata (choice A ). The prognosis is generally good in benign metastasizing leiomyomata; however, it carries greater mortality and morbidity than disseminated peritoneal leiomyomatosis.

\section{REFERENCES}

1. K urman RJ. Blaustein's Pathology of the female genital tract (4th ed). Springer; N ew Y ork, B erlin 2002; pp 497-98.

2. V aquero M E, M agrina J F, L eslie K $O \mathrm{~J}$. U terine smooth-muscle tumors with unusual grow th patterns. M inim Invasive Gynecol 2009;16(3):263.

3. $Y$ amaguchi $T$, I mamura $Y, Y$ amamoto $T$, Fukuda $M$. L eiomyomatosis peritoneal is disseminata with malignant change in a man. Pathol Int 2003;53:179-85.

4. Fujii S, Nakashima N, O kamura H, Takenaka A, Kanzaki H, O kuda Y, et al. Progesterone-induced smooth muscle-like cells in the subperitoneal nodules produced by estrogen. Experimental approach to leiomyomatosis peritoneal is disseminata. A m J Obstet Gynecol 1981;139(2):164-72.

5. Drake A, Dhundee J, Buckley CH, Woolas R. Disseminated leiomyomatosis peritonealis in association with oestrogen secreting ovarian fibrothecoma. Brit J Obstet Gynaecol 2001;108:661-64.

6. B ristow RE, M ontz FJ . L eiomyomatosis peritonealis disseminata and ovarian B renner tumour associated with tamoxifen use. Int J Gynecol Cancer 2001;11:312-15.

7. B ekkers RLM , W illemsen WNP, Schijf CPT, et al. Leiomyomatosis peritonealis disseminata: D oes malignant transformation occur? A literature review. Gynecol Oncol 1999; 75(1):158-63.

8. Raspagliesi F, Quattrone P, Grosso G, Cobellis L, Di Re E. $M$ alignant degeneration in leiomyomatosis peritonealis disseminata. Gynecol Oncol 1996; 61(2):272-74.

9. Hales HA, Peterson CM, Jones K P, Quinn JD. L eiomyomatosis peritonealis disseminata treated with a gonadotropin-releasing hormone agonist. A case report. A m J Obstet Gynecol 1992;167(2):515-16.

10. Fulcher AS, Szucs RA . L eiomyomatosis peritonealis disseminata complicated by sarcomatous transformation and ovarian torsion: Presentation of two cases and review of the literature. A bdom Imaging 1998;23(6):640-44.

\section{ABOUT THE AUTHORS}

\section{Ulrich Honemeyer}

Specialist, A ssociateProfessor, O bstetricsand Gynecology, Department of M other and Child W elcare Hospital, Dubrovnik International University, Dubai, UAE

\section{J ason R Ross}

Student Physician, Department of M edical Education, Paul L Foster School of M edicine, Texas Tech U niversity, EI Paso, Texas, USA 


\section{J ami J Barnard}

Student Physician, Department of M edical Education, Paul L Foster School of M edicine, Texas Tech U niversity, EI Paso, Texas, USA

\section{Kallie Appleton}

Student Physician, Department of M edical Education, Paul L Foster School of M edicine, Texas Tech U niversity, EI Paso, Texas, USA

\section{Thomas G Tullius J $r$}

Student Physician, Department of Medical Education, Paul L Foster School of M edicine, T exas T ech U niversity, EI Paso, Texas USA

\section{Lisa Montgomery}

Student Physician, Department of Medical Education, Paul L Foster School of Medicine, Texas Tech University, EI Paso Texas, USA

\section{Sanja Kupesic Plavsic (Corresponding Author)}

Professor, D epartment of Obstetrics/G ynecology and R adiology, Paul L Foster School of M edicine, Texas Tech University, 4800 A lberta A ve, EI Paso, Texas 79905, USA, Phone: +1 (915)783-1700 e-mail: sanja.kupesic@ ttuhsc.edu 